\title{
A FLEXIBLE MEMS TECHNOLOGY AND ITS FIRST APPLICATION TO SHEAR STRESS SENSOR SKIN
}

\author{
Fukang Jiang, Yu-Chong Tai, Ken Walsh, Tom Tsao, Gwo-Bin Lee* and Chih-Ming Ho* \\ Department of Electrical Engineering \\ California Institute of Technology \\ Pasadena, CA 91125, USA \\ *Department of Mechanical and Aerospace Engineering \\ University of California \\ Los Angeles, CA 90095, USA
}

\begin{abstract}
A new microfabrication technology that enables the integration of MEMS devices on a flexible polyimide skin has been developed. Mechanically, the flexible skin consists of many individual Si islands (necessary for silicon MEMS/electronics devices) that are connected together by a thin/thick polyimide film (typically 1-100 $\mu \mathrm{m}$ thick). To create the islands, Si diaphragms are first formed with a desirable thickness $(10-500 \mu \mathrm{m})$ by Si wet etching and then patterned from the back side by reactive ion etching (RIE). As a first application, flexible shearstress sensor skins for aerodynamics study have been fabricated. The finished skin is $3 \mathrm{~cm}$ long and $1 \mathrm{~cm}$ wide, and it consists of about 100 sensors. The skin polyimide is $17 \mu \mathrm{m}$ thick and the silicon islands are $75 \mu \mathrm{m}$ thick. These skins have been successfully taped on a semicylindrical ( $1.3 \mathrm{~cm}$ diameter) delta wing leading edge to perform real-time 2-D shear stress profiling.
\end{abstract}

\section{INTRODUCTION}

For some applications, it is essential to obtain the realtime 2-D profiling of certain physical parameters such as temperature, force, pressure or shear stress on a 3-D object. If the surface of the object is flat, this profiling can be achieved by using a monolithic MEMS device with a large amount of sensors [1]. However, this is a lot more difficult if the surface is non-planar. For example, in aerodynamics study, the most popular research objects such as an air foil have non-planar or even highcurvature surfaces. In the past, if real-time distribution measurement was necessary, embedding all the discrete sensors on a surface was the only way. However, large sensor size and difficulty in packaging, i.e., plumbing and wiring, have long been the limiting factors to good measurements.

It has been our goal to develop a flexible MEMS tech- nology to produce smart skins (with integrated MEMS devices) that can be easily taped or glued on non-planar surfaces. Retrospectively, Barth et al. [2] in 1985 reported the first version of this idea with a onedimensional flexible Si-diode temperature sensor array in which a polyimide strip was the flexible material connecting $\mathrm{Si}$ islands formed by isotropic HNA etching. However, the authors claimed that this technology needs some major improvements before it can be applied to more complicated sensor system. This sensor skin concept, unfortunately, was not pursued further until 1994, when Beebe and Denton [3] presented their effort on improving the robustness and reliability of flexible polyimide skins which do not have any device. The major lead failure mechanism was identified as the breakage of the thin silicon on the island periphery. Since the basic process was the same as that developed by Barth, the methods used to enhance the robustness, including the application of tape and coating of epoxy on both front and back sides of the skins, were all performed manually as post-processing steps. It is obvious that these are not the ideal solution for a reliable as well as massproducible smart skin technology. Just recently, we learned that, almost concurrent to our effort, Bang and Pan [4] also has an on-going project to develop a flexible heat-flux sensor array which is made by direct deposition of thin-film metals on commercial Kapton substrates. A large array of metal temperature sensors can be made in this way but, its drawback is that neither ICs nor silicon MEMS can be integrated with this approach; hence, only limited types of sensors are available and hybrid assembly of electronic circuits is unavoidable.

In comparison, the work we present here is a new flexible skin technology designed to be compatible with both IC and MEMS fabrications. Due to the much stronger periphery of the silicon islands formed by nearly vertical RIE, the skin reliability has been greatly improved. Moreover, we have realized a 2-D flexible skin integrated with more than 100 shear stress sensors. This skin 
has further been mounted on a 3-D leading edge of a delta wing and successful 2-D shear stress measurement is obtained. For the first time, the air flow boundary layer separation over the leading edge of a delta wing is determined experimentally in real time.

\section{FLEXIBLE SKIN TECHNOLOGY}

As mentioned in the previous section, almost all the lead failures on the flexible skins made by Barth and Beebe were caused by the breakage of the thin peripheries on the Si island during the squeezing and folding test. The thin and weak Si island periphery was the natural result of isotropic HNA etching (Fig. 1a). In comparison, the island shapes formed by caustic anistropic etchants such as TMAH or $\mathrm{KOH}$ and by the combination of anisotropic etching and RIE are much more robust when subjected to squeezing and folding (Fig. $1 \mathrm{~b} \& \mathrm{c}$ ). Unfortunately, the above caustic etchants attack all types of polyimides, so that polyimide layers must be coated after the islands are formed or protected from the etchants during the etching. Using gold or other expensive metals as the protection materials is not only costly, but also incompatible with IC process. The one-sided etching apparatus is not usable as the pressure difference between the two side would cause the rupture of the polyimide near the end of the etching, or a small leak on the wafer near the end of the etching would attack the polyimide on the front side. The former approach is not feasible either, unless proper material is remained to support the islands after the

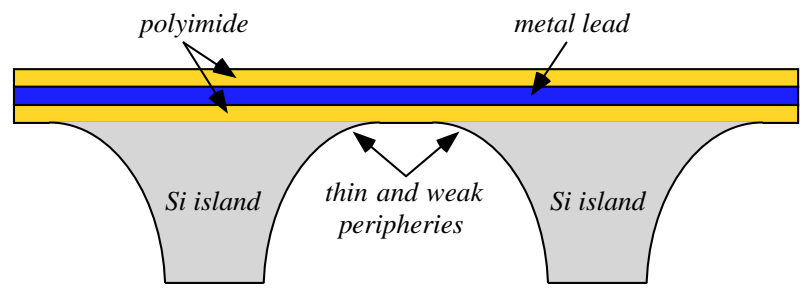

(a) by HNA isotropic etching.

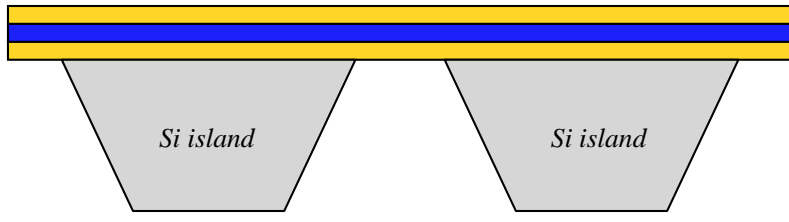

(b) by anisotropic etching (TMAH or $\mathrm{KOH}$ ).

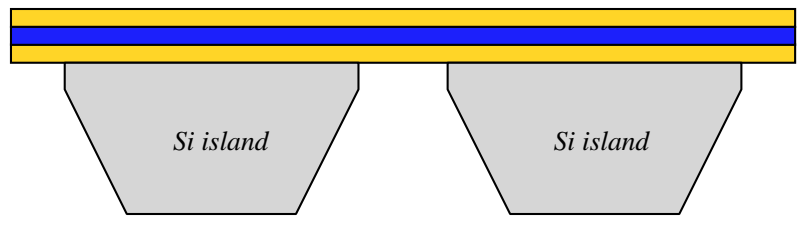

(c) by a combination of anisotropic etching and RIE.

Fig. $1 \mathrm{Si}$ island shapes formed by different ways. etching and can be removed with ease after polyimide is coated. Our new flexible MEMS technology is based on this idea with additional modification.

1. TMAH or $\mathrm{KOH}$ selectively etches backside.

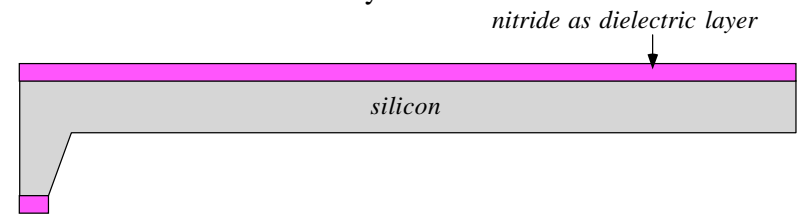

2. Aluminum/polyimide/aluminum/polyimide processing on frontside.

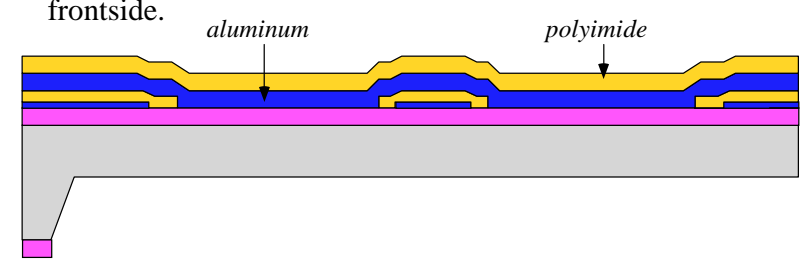

3. RIE etches backside using Al mask. Polyimide processing on backside.

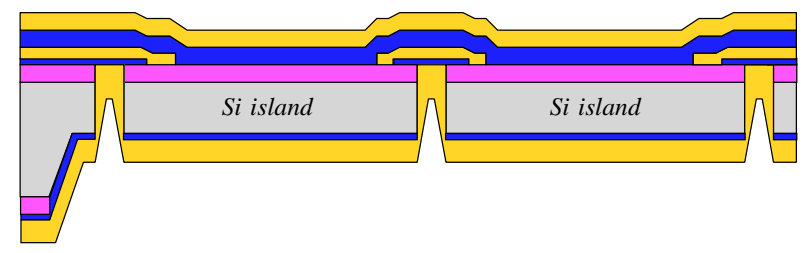

Fig. 2 Simplified process flow of the new flexible skin technology

Fig. 2 is the simplified process flow of this new technology. It starts with selective TMAH or $\mathrm{KOH}$ etching on the backside of a Si wafer using silicon nitride as a mask to form a Si membrane of desired thickness. Aluminum is evaporated on the front side and patterned to cover the area between future $\mathrm{Si}$ islands. Polyimide is spun-on, cured and patterned to cover the protective $\mathrm{Al}$ completely. Normal Al metallization then follows to provide electrical leads. Another polyimide layer is spun-on and patterned to expose the bonding pads. RIE etching on the backside using $\mathrm{Al}$ as masking material removes the $\mathrm{Si}$ on the streets between $\mathrm{Si}$ islands. Here the first layer of $\mathrm{Al}$ serves as the etch stop in the $\mathrm{SF}_{6}$-based RIE etching. Finally, a thick polyimide layer is spun-on and cured on the backside to sandwich the $\mathrm{Si}$ islands. The finished skins are then cut off from the Si wafer frame by a razor blade. Fig. 3 shows the photograph of a fabricated flexible skin about $8 \mathrm{~cm}$ in diameter with $2 \times 2 \mathrm{~mm}^{2} \mathrm{Si}$ islands. Since we are only demonstrating the concept here, the skin did not contain actual devices. However, it is obvious that the above process is compatible with IC process as it involves only aluminum and polyimide which are commonly used in IC fabrication. We can start with a wafer with fabricated IC and MEMS (without metallization) and the above process needs only minimal adjustment to produce flexible MEMS skins. Such flexi- 


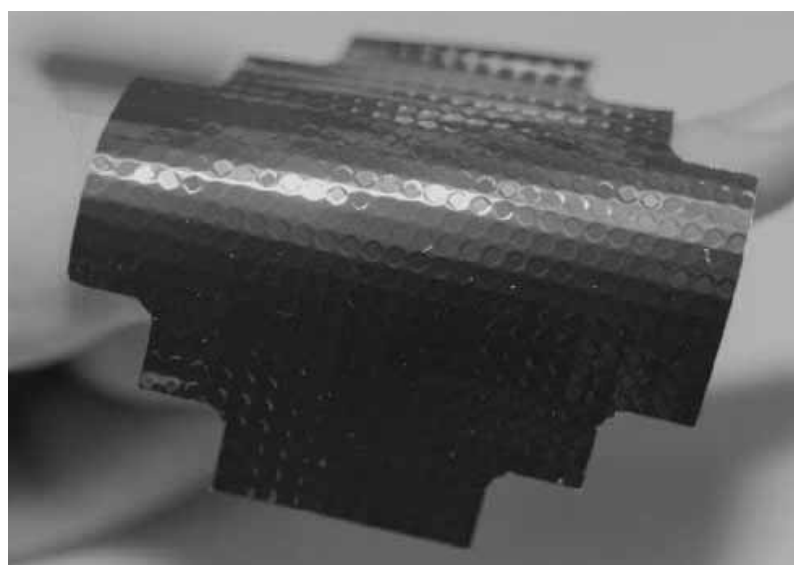

Fig. 3 Picture of a wafer-size flexible skin.

ble MEMS skin has already been realized and will be presented later in this paper.

In the above process, the $\mathrm{Si}$ islands are formed by RIEetching the Si diaphragm. Since the diaphragm thickness is less than $100 \mu \mathrm{m}$ and the RIE etching is nearly vertical, we can well-define islands with dimension as small as $100 \mu \mathrm{m}$ and spacing less than $50 \mu \mathrm{m}$, which makes it possible for the skin to be applied on a very highcurvature surface with good conformal coverage. This can not happen if any of the island shaping techniques shown in Fig. 1 is used for some obvious reasons (HNA etching is isotropic and TMAH or $\mathrm{KOH}$ etching requires large corner compensation structures to avoid fast undercut on corners). Of course, there is a disadvantage in using $\mathrm{Si}$ diaphragm and islands. First, care must be taken on the wafers during processing as they are very fragile. However, if the individual skin is not too big, the wafers are reasonably robust to survive the whole process. Second, thin $\mathrm{Si}$ islands can not take as much force as thick ones.

Due to its good mechanical strength and flexibility, polyimide was chosen as flexible skin material, just as in the previous works. We used DuPont's PI-2808 polyimide which has a tensile strength of $210 \mathrm{MPa}$ [5]. This value is one of the highest among all the spin-coated polyimides and is almost the same as that of Kapton (231 $\mathrm{MPa}$ ) [6], a product also made by DuPont and commonly used as a flexible runner for providing reliable connections to moving print heads. In the process, the thickness of each polyimide layers on the front side of the wafer are $3-4 \mu \mathrm{m}$ after cure at $350^{\circ} \mathrm{C}$. Since all the polyimide layers have not been exposed to strong acids or bases, their mechanical properties are not degraded. Then a total of $7 \mu \mathrm{m}$ thick polyimide on the front should be able to stand a tensile force of $1.47 \mathrm{~kg} / \mathrm{mm}$, which is fairly strong. However, the peel-off forces of the polyimide from $\mathrm{Si}$ substrates given by the manufacturer is only about $0.23 \mathrm{~g} / \mathrm{mm}$. Therefore, even a very small shear force exerted on a Si island would peel it off. One solution to this problem is to spin thick polyimide (10 $\mu \mathrm{m})$ on the back side of the wafer to fully encapsulate the islands. The total polyimide thickness between islands is now $17 \mu \mathrm{m}$ and the maximum tensile force the skin can stand is increased to $3.57 \mathrm{~kg} / \mathrm{mm}$.

As for the metal leads, they are completely embedded in two polyimide layers on the flexible area. Also, the Si islands they are sitting on do not have weak edges. Therefore, they should be able to stand repetitive squeezing and bending without breakage. In our initial testing, no metal lead failure has been observed after more than 100 times of $90^{\circ}-180^{\circ}$ bending.

\section{FLEXIBLE SHEAR STRESS SENSOR ARRAY}

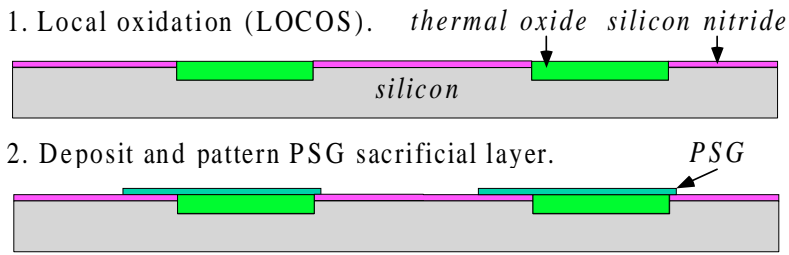

3. Deposit nitride diaphragm and open etch holes.

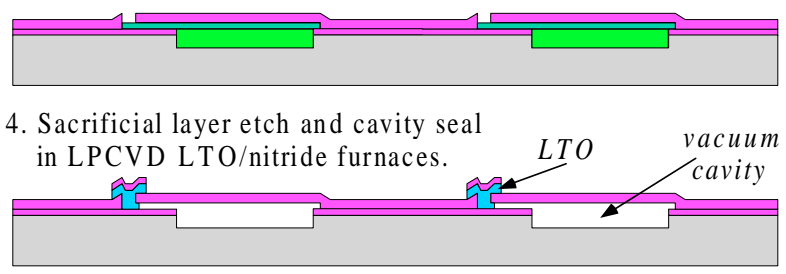

5. Deposit, dope and pattern polysilicon. Deposit thin nitride for passivation.

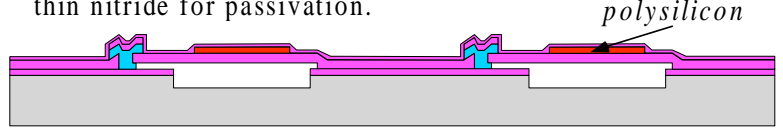

6. TMAH or $\mathrm{KOH}$ etches backside (front side protected with LTO/nitride).

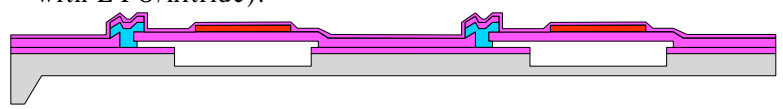

7. Aluminum/polyimide processing + contact opening + aluminum/polyimide processing on frontside.

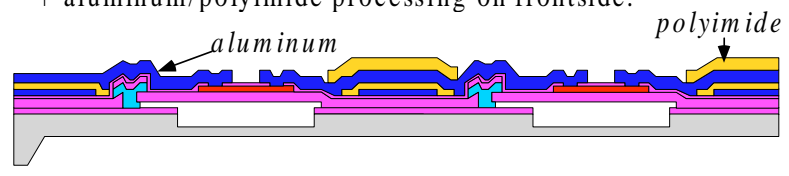

8. RIE etches backside using Al mask. Polyimide processing on backside.

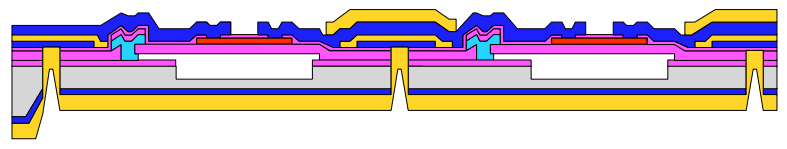

Fig. 4 Fabrication process flow of the flexible shear stress sensor skin. 
It is important to demonstrate that useful MEMS devices can indeed be integrated on flexible substrate with this flexible skin technology. Based on the following two considerations, we target our first such effort on the development of a flexible shear stress sensor array. First, the shear stress sensor (a vacuum-insulated diaphragmtype thermal sensor capable of measuring the wall shear stress exerted by viscous flow) is useful in turbulent flow study since its invention. For example, arrays of shear stress sensor on a rigid substrate have been used to detect the real-time shear stress distribution on a flat (i.e., 2-D) surface for flow-induced drag reduction study [1] [7]. However, its application has so far been limited to 2D flow due to the lack of flexible packages. The development of a flexible shear stress sensor array is the only way to extend its application to 3-D flow. Second, the fabrication of shear stress sensors is a surface micromachining process with reasonably high complexity. It will be a good demonstration of the compatibility between the flexible skin and MEMS technologies.

Fig. 4 shows the complete fabrication process flow of the flexible shear stress sensor array, which is basically the combined fabrication processes for the shear stress sensor and the skin. The only mixing is that contacts are opened after the first aluminum/polyimide processing so that they are fresh and clean for the following metallization. Figs. 5 and 6 are the photographs of a shear stress sensor and the finished flexible sensor respectively. The skin is $1 \mathrm{~cm}$

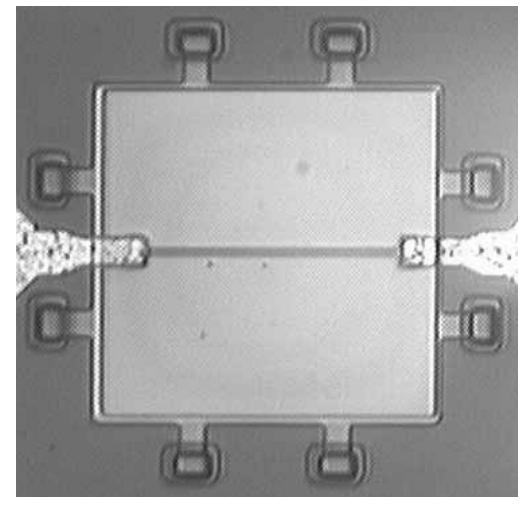

Fig. 5 Photograph of a shear stress sensor. A square silicon nitride diaphragm $\left(200 \times 200 \mu \mathrm{m}^{2}\right)$ with an embedded polysilicon wire is on top of a vacuum-sealed cavity.

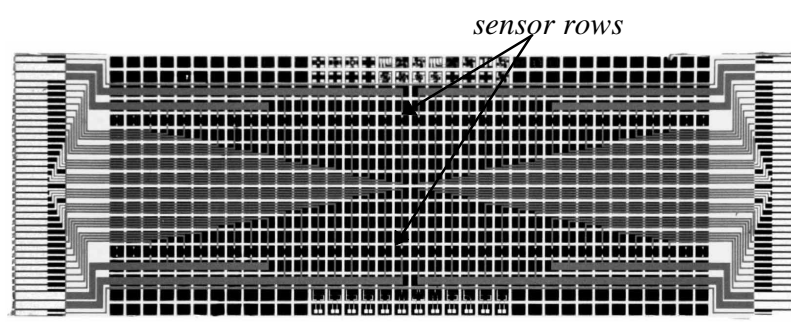

Fig. 6 Picture of a flexible shear stress sensor array $(1 \mathrm{~cm} \times 3$ $\mathrm{cm})$.

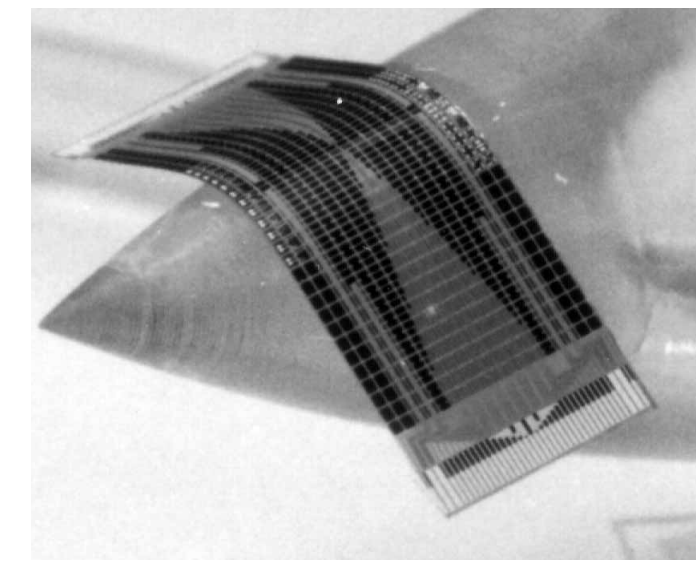

Fig. 7 A flexible shear stress sensor skin sits on a conic object. The bending is caused by gravitation.

wide, $3 \mathrm{~cm}$ long, and it consists of two 32 -sensor rows with a horizontal pitch of $635 \mu \mathrm{m}$ and many other test devices (a total of more than 100 sensors). The two sensor rows are $5 \mathrm{~mm}$ apart and located between the pairs of white square boxes in the picture. Their bonding pads are extended to the left and right edges of the skin. The layout is designed in such a way that each sensor row spans the semi-cylindrical surface $(1.3 \mathrm{~cm}$ in diameter $)$ of a delta wing leading edge under study with an angular resolution of $5.6^{\circ}$. Each sensor occupies $250 \times 250 \mu \mathrm{m}^{2}$ and the dimension of each $\mathrm{Si}$ island is $450 \mu \mathrm{m} \times 550 \mu \mathrm{m}$ (75 $\mu \mathrm{m}$ thick) to fully accommodate one sensor and to achieve excellent surface smoothness and conformability. Fig. 7 shows the picture of a skin sitting on a conic object. Its good flexibility is seen from the bending of the skin due to gravitation.

\section{CALIBRATION, PACKAGING AND TESTING}

To test the shear stress sensor skin, it is first flushmounted on a wind-tunnel where controlled shear flow is available for sensor calibration. The sensors on the flexible skin have been found to behave the same as those on rigid substrates. This can be seen from the calibration results shown in Fig. 8, where the square of the output voltage is proportional to the one-third power of shear stress $\tau$ and the sensitivity is about $100 \mathrm{mV} / \mathrm{Pa}$ under constant temperature bias. Next, the skins are mounted on one of the two 3-D leading edges of a delta wing (Fig. 9). The leading edge is divided into many blocks of removable semi-cylinders $2 \mathrm{~cm}$ long and $1.3 \mathrm{~cm}$ in diameter. The sensor block is recessed by about $100 \mu \mathrm{m}$ to compensate the skin thickness. At this moment, we use only one row of sensors to measure the instantaneous distribution of shear stress on that location. By moving the skins along the whole leading edge block by block, we are able to map out the steady-state shear stress distribution. 


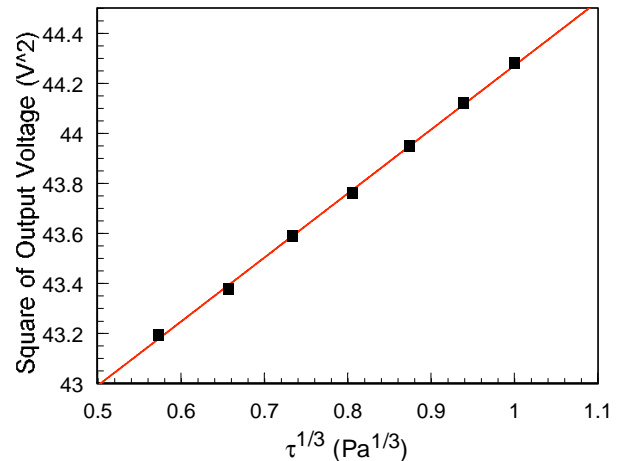

Fig. 8 Calibration curve of a sensor on a flexible skin mounted on a flat surface. The sensor is biased in constant temperature mode.

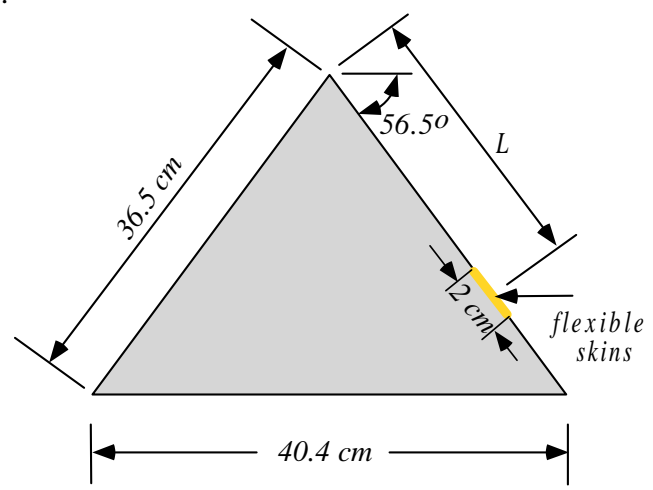

Fig. 9 Schematic of the delta wing.

Fig. 10 shows the schematic of the cross section of our packaged sensor block for delta wing test. First, two skins are glued to the semi-cylinder with the sensors on the curved surface and the bonding leads/pads extended to the flat surface (Fig. 11). Then a circuit board with pre-soldered wires is attached to the flat surface next to the bonding pads of the skins. Ultrasonic wire bonding is performed to connect the leads to the circuit board and then the bonding wires are fixed by epoxy. Here, the circuit board is a piece of Si with gold bonding and soldering pads specially designed and fabricated for this purpose (Fig. 12). The finished delta wing is then shown in Fig. 13, which is ready for wind-tunnel testing.

Measurements have been done under different flow velocities $(U)$, skin locations $(L)$ and angles of attack (AOA, defined as the angle between the air flow and the delta wing plane). For example, Fig. 14 shows the averaged output voltages (after gain of 10) of the sensors for $U=30 \mathrm{~m} / \mathrm{s}, L=29 \mathrm{~cm}$ and $A O A=30^{\circ}$. Sensor locations are indicated by $\theta$, which is $0^{\circ}$ at the bottom surface and $180^{\circ}$ at the top surface. The averaged shear stress has a minima at about $110^{\circ}$, which is a result of flow over cylinder [8-9]. It is also non-trivial to determine a flow separation point, where the flow boundary layer starts to separate from the leading edge surface. Typically, the surface shear stress fluctuation stays low before separation, and rises sharply after separation. Therefore, we can identify a separation point through the measurement of the root-mean-square (RMS) value of shear stress fluctuation. Fig. 15 shows the RMS results of one measurement, from which the separation point is found to be at $\theta$

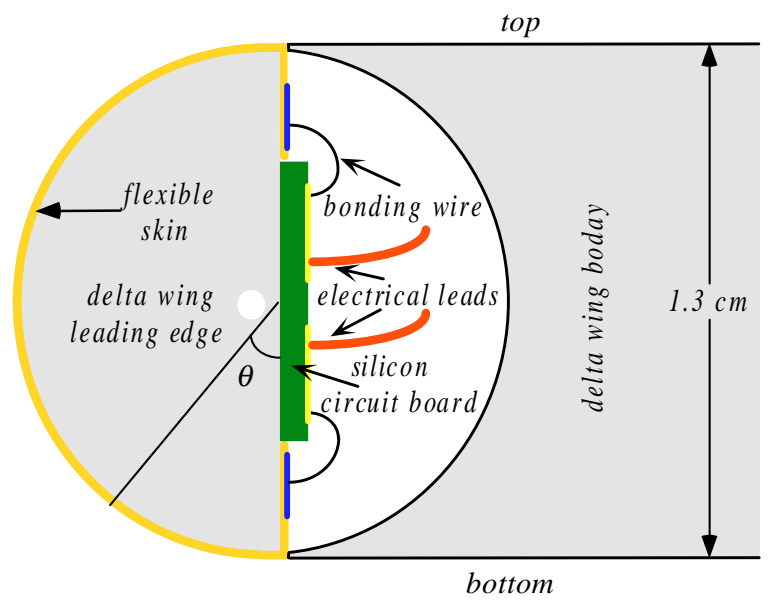

Fig. 10 Packaging scheme for the flexible shear stress sensor skin on delta wing edge.

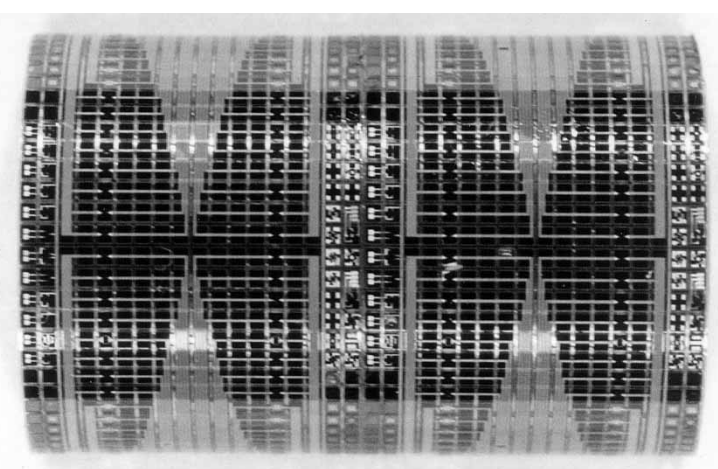

Fig. 11 Two flexible skins wrapped around on a semicylindrical block, $2 \mathrm{~cm}$ long and $1.3 \mathrm{~cm}$ in diameter.

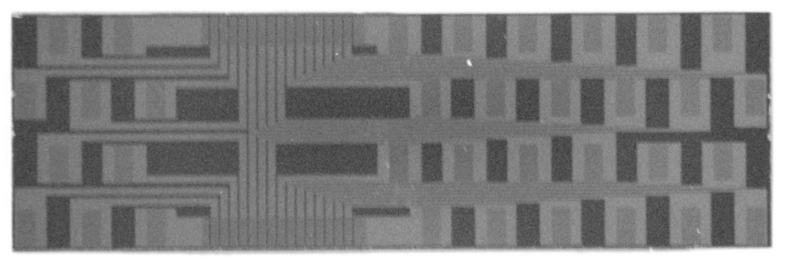

Fig. 12 Picture of a Si circuit board.

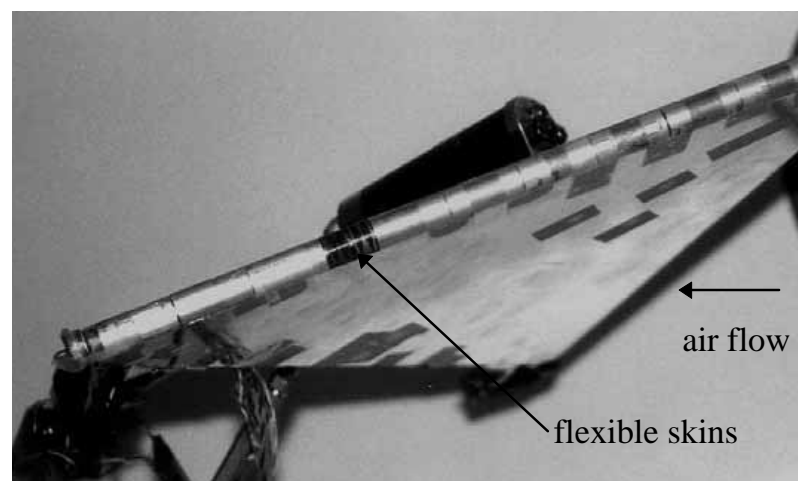

Fig. 13 Picture of the skins-mounted delta wing model. 
$=80^{\circ}$. In fact, this is the first time separation points are experimentally determined in real time. The separation line along the leading edge is consistent with the data measured from a single shear stress sensor that was placed around the leading edge point by point in steady state flow (Fig. 16) [10]. Based on our data, it is concluded that, for real time flow control, a single sensor is no longer enough and the flexible shear stress arrays necessary because the flow separation point along the leading edge is a function of changing $U, L$ and $A O A$ in real flow field.

\section{CONCLUSION}

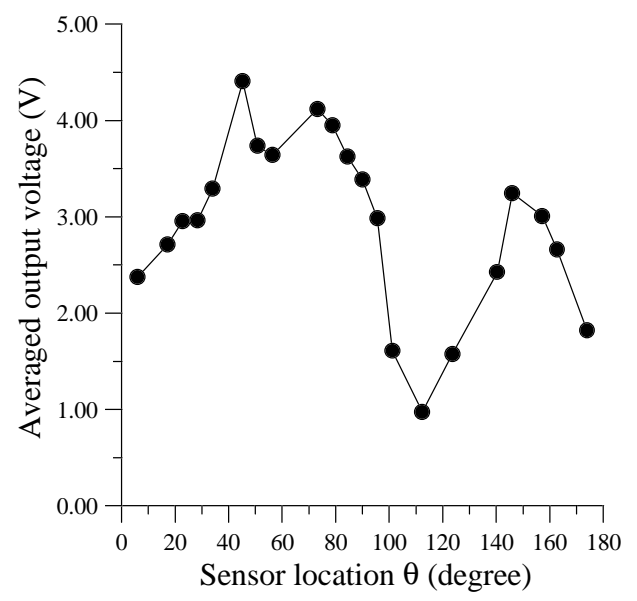

Fig. 14 Averaged output from one row of sensors on a skin.

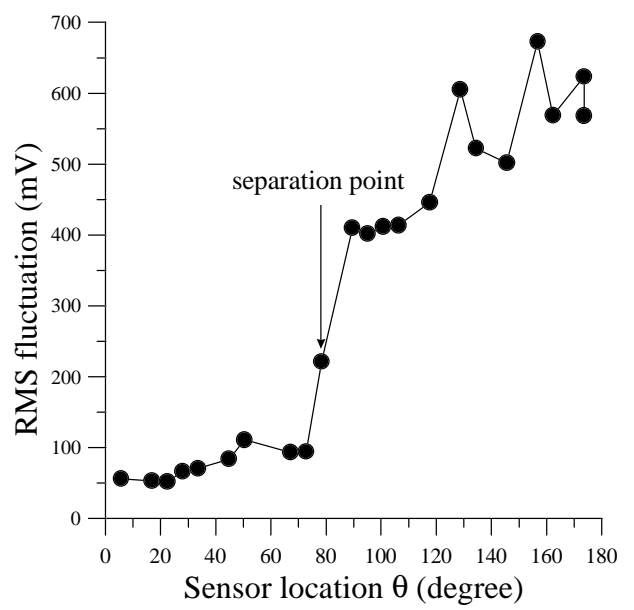

Fig. 15 RMS fluctuation used to identify separation point.

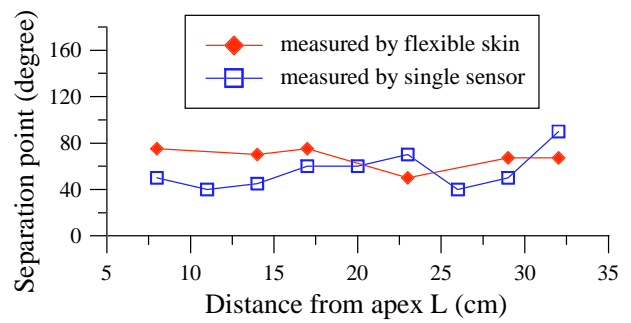

Fig. 16 Comparison of separation lines measured by a single sensor and a flexible shear stress sensor array.
A novel flexible MEMS skin technology has been developed and it is fully compatible with IC process. The major lead failure occurred in previously reported technologies has been eliminated through the proper shaping of $\mathrm{Si}$ islands. Moreover, $\mathrm{Si}$ islands as small as $100 \mu \mathrm{m}$ can be defined with good accuracy, which allows the skins to be applied on small surfaces with large curvatures. The first application of this technology has produced a flexible shear stress sensor array that was used in aerodynamics for the real-time measurement of shear stress distribution on 3-D surfaces.

\section{ACKNOWLEDGMENTS}

This work is supported by DARPA and AFOSR under grant \#F49620-94-1-0008.

\section{REFERENCES}

[1] F. Jiang, Y.C. Tai, B. Gupta, R. Goodman, S. Tung, J.B. Huang and C.H. Ho, "A Micromachined Shear Stress Sensor Array", Proc. IEEE MEMS-96 Workshop, San Diego, pp. 110-115, 1996.

[2] P.W. Barth, S. L. Bernard and J. B. Angell, "Flexible Circuit and Sensor Arrays Fabricated by Monolithic Silicon Technology", IEEE Trans. Electron. Devices, vol. ED-32, no. 7, pp. 1202-1205, 1985.

[3] D. J. Beebe and D. D. Denton, "A Flexible Polyimide-Based Package for Silicon Sensors", Sensors and Actuators, Vol. A44, pp. 57-64, 1994.

[4] C. Bang and T. Pan, "Flexible Heat Flux Sensor Arrays", AFOSR Contractor and Grantee Meeting on Turbulence and Internal Flows, Atlanta, Georgia, 4-6 Sept. 1996.

[5] DuPont Electronic Materials, PI-2808 Polyimide Product Information, 1994.

[6] DuPont, Kapton Polyimide film Productor Information.

[7] B. Gupta, R. Goodman, F. Jiang, Y. C. Tai, S. Tung and C. H. Ho, "Analog VLSI System for Active Drag Reduction", IEEE Micro, Vol. 16, No. 5, pp. 53-59, 1996.

[8] B. J. Bellhouse and D. L. Schultz, "Determination of Mean and Dynamic Skin Friction, Separation and Transition in Low-Speed Flow with a Thin-Film Heated Element", J. Fluid Mech., Vol. 24, part 2, pp. 379-400, 1966.

[9] Elmar Achenbach, "Total and Local Heat Transfer from a Smooth Circular Cylinder in Cross-Flow at high Reynolds Number", Int. J. Heat Mass Transfer, Vol. 18, pp. 1387-1396, 1975.

[10] G. B. Lee, C. M. Ho, F. Jiang, C. Liu, T. Tsao and Y. C. Tai, "Distributed Flow Control by MEMS", ASME 1996 International Mechanical Engineering Congress and Exposition, Atlanta, Geo, Nov. 17-22, 1996. 